
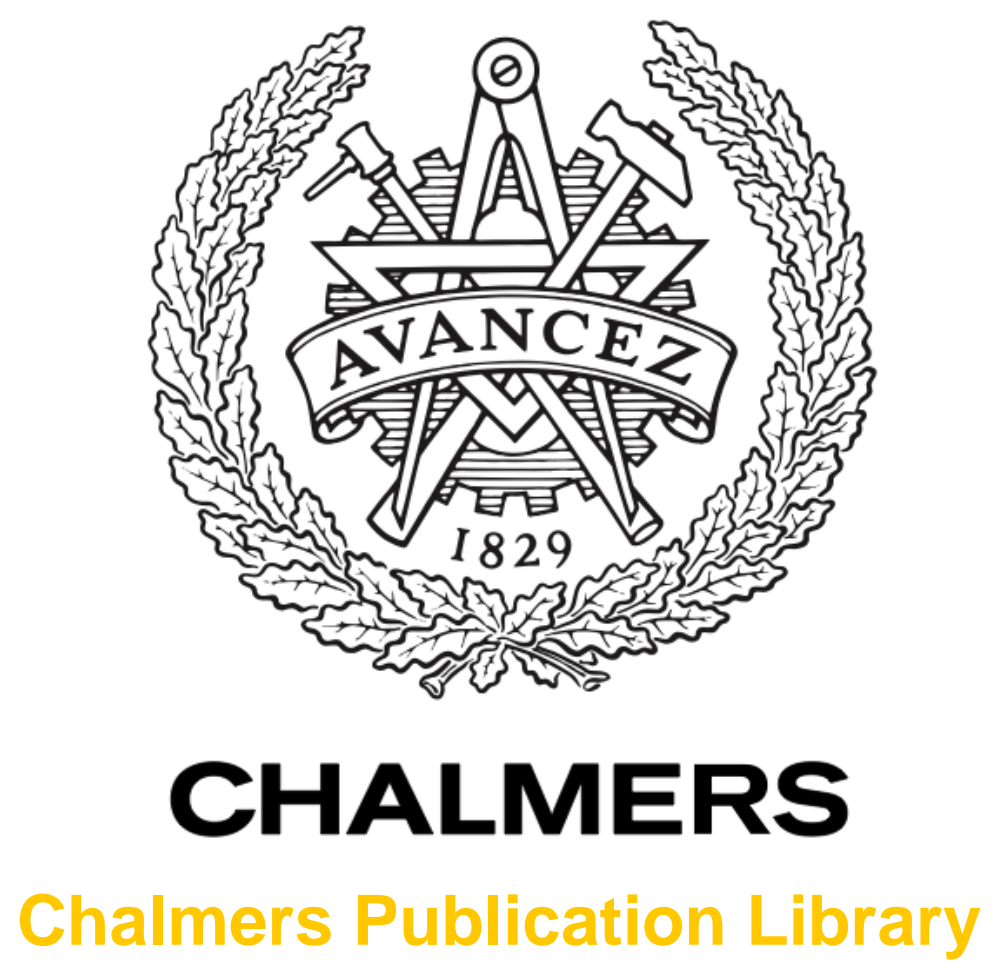

Low speed maneuvering assistance for long vehicle combinations

This document has been downloaded from Chalmers Publication Library (CPL). It is the author's version of a work that was accepted for publication in:

IEEE Intelligent Vehicles Symposium, Proceedings

Citation for the published paper:

Hoel, C. ; Falcone, P. (2013) "Low speed maneuvering assistance for long vehicle combinations". IEEE Intelligent Vehicles Symposium, Proceedings pp. 598-604.

http://dx.doi.org/10.1109/IVS.2013.6629532

Downloaded from: http://publications.lib.chalmers.se/publication/193519

Notice: Changes introduced as a result of publishing processes such as copy-editing and formatting may not be reflected in this document. For a definitive version of this work, please refer to the published source. Please note that access to the published version might require a subscription. 


\title{
Low Speed Maneuvering Assistance For Long Vehicle Combinations
}

\author{
Carl-Johan Hoel and Paolo Falcone.
}

\begin{abstract}
This paper considers a low speed maneuvering problem for long articulated vehicle combinations. High precision maneuvering is achieved by designing a model-based state feedback optimal control method, commanding the steering of the first unit and a moveable coupling point between the first unit and the trailer. Simulation results are presented for a tight 90 degree turn, involving both forward and backward motions.
\end{abstract}

\section{INTRODUCTION}

Professional drivers of heavy trucks with trailers often have to perform low speed maneuvers with high precision. Examples are when loading or unloading the vehicle, docking at ramps, parking in tight spaces, coupling trailers or changing swap bodies. Even for experienced drivers, such high precision maneuvering can be a difficult task, which is both time consuming and can be hazardous.

This paper considers a maneuvering problem for a Nordic Combination $^{1}$, consisting of a truck with a dolly and a semitrailer, see Fig. 1. However, the proposed approach can be extended to other combinations as well. The automated execution of the considered maneuvers is a complex task that can be decomposed into several problems, e.g. sensing, path planning and motion control. This paper focus on motion control, while briefly mentioning path planning and omitting the sensing task.

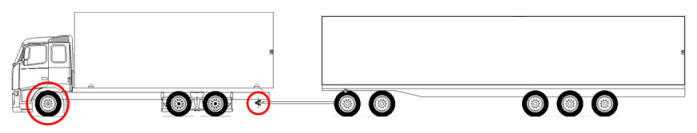

Fig. 1. The Nordic Combination; $25.25 \mathrm{~m}$ long combination consisting of a truck with a dolly and a semitrailer. The two actuators, controlling the steering angle and lateral coupling point position, are highlighted.

In literature many different control strategies for reversing an articulated vehicle have been proposed. The vehicle nonholonomicity, constraining its motion capabilities, and the system instability make the reversing problem challenging. A viable approach, presented in, e.g., [1] is to linearize the system and then apply linear control theory, such as pole placement or Linear Quadratic (LQ) control thus achieving local asymptotic stability. Input-output linearization, presented in [3], can be performed by designing a control

C. Hoel is with the Department of Chassis Strategies \& Vehicle Analysis, Volvo Group Trucks Technology, 40508 Göteborg, Sweden (e-mail: carl-johan.hoel@volvo.com)

P. Falcone is with the Department of Signals and Systems, Chalmers University of Technology, 41296 Göteborg, Sweden (e-mail: paolo.falcone@chalmers.se)

${ }^{1}$ Called Nordic Combination because it is common in the Nordic countries, where the maximum allowed vehicle combination length is $25.25 \mathrm{~m}$, as opposed to most of Europe where the maximum length is $18.75 \mathrm{~m}$. law that cancels the nonlinear parts of the system, thus transforming it into a linear system. Then linear control theory can be applied. A completely nonlinear approach is presented in [2], based on Lyapunov techniques. Neural networks [7] and fuzzy controllers [6] have also been used for this class of problems. Exact linearization has been proposed [9], but this is only applicable for on-axle hitching, which is not the case considered in this paper.

The solution strategy applied in this paper is a controller based on LQ feedback together with feed-forward action. The main contribution is the development of a speed independent vehicle model, expressing the vehicle state as an offset from a nominal state trajectory. Furthermore, a special approximation is introduced to find the nominal trajectory from a geometric path in an euclidean space. To relax the nonholonomic constraints of the vehicle, a new motion actuator is suggested. This actuator moves the coupling point at the rear of the truck in the lateral direction. Then it is possible to steer the trailer somewhat independently of the truck. Fig. 1 highlights the used actuators. The coupling actuator also has other advantages, such as allowing reduced swept path width during cornering and reduced rearward amplification of yaw rate (not treated in this paper), motivating the additional cost.

The paper is organized as follows. Section II describes how the vehicle is modelled. Then in Section IV an offset model from the desired path is derived and linearized, followed by an outline of the control strategy. Simulation results are shown in Section V. The paper ends with some concluding remarks in Section VI.

\section{VEHICLE MODELLING}

Two vehicle models are used in this paper. First a kinematic bicycle model is used for derivation of the control algorithms. Then a complex vehicle model, called Virtual Truck Model (VTM), is used for evaluation of controller performance. VTM is an advanced vehicle dynamics model developed and used at Volvo Group Trucks Technology. It models the vehicle dynamics in Simulink and SimMechanics, using for example the PAC2002 Magic Formula tyre model and including effects generated by the frame torsion. The model has been validated against real test data and proved to be accurate.

\section{A. Kinematic model}

Since low speed reversing maneuvers are considered in this paper, the roll and pitch motions of the vehicle are ignored. All the wheels of each axle are lumped together into one wheel in the middle, as in, e.g., [8]. Moreover, multiple axle groups can be approximated by a single axle for each 
group, with an equivalent wheelbase as in, e.g., [10]. Thereby the number of axles of the Nordic Combination is reduced from 8 to 4 . Finally, the vehicle is assumed to move without skidding sideways, i.e., without lateral slip. This assumption is deemed reasonable because of the low speed.

Fig. 2 shows the reduced model of the Nordic Combination, including the variables used in the rest of the section.

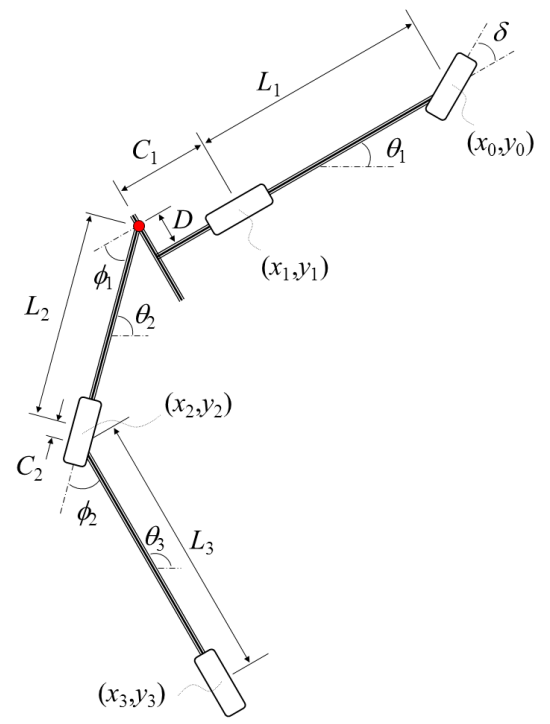

Fig. 2. Bicycle model of a Nordic Combination (truck - dolly - semitrailer).

A reference point, i.e., the point that should track the path, is introduced at the rear axle of the semitrailer. The states are the position of the reference point $x_{3}, y_{3}$, the yaw angle of the trailer $\theta_{3}$, the two articulation angles $\phi_{1}$ and $\phi_{2}$, the steering angle $\delta$ and the lateral coupling position $D$. The system has two control inputs, steering angle rate $u_{1}$ and coupling speed $u_{2}$. Moreover, the speed of the truck $v_{1}$, which is controlled by the driver, is considered as a model parameter.

The state space model is

$$
\begin{aligned}
\dot{x}_{3} & =v_{3} \cos \theta_{3}, \\
\dot{y}_{3} & =v_{3} \sin \theta_{3}, \\
\dot{\theta}_{3} & =v_{1}\left(-\frac{1}{L_{3}} \cos \phi_{1} \sin \phi_{2}+\frac{C_{1} \sin \phi_{1}+D \cos \phi_{1}}{L_{1} L_{3}} \tan \delta \sin \phi_{2}\right. \\
& \left.+\frac{C_{2}}{L_{2} L_{3}} \sin \phi_{1} \cos \phi_{2}+\frac{C_{2}\left(C_{1} \cos \phi_{1}-D \sin \phi_{1}\right)}{L_{1} L_{2} L_{3}} \tan \delta \cos \phi_{2}\right) \\
& -u_{2}\left(\frac{1}{L_{3}} \sin \phi_{1} \sin \phi_{2}+\frac{C_{2}}{L_{2} L_{3}} \cos \phi_{1} \cos \phi_{2}\right), \\
\dot{\phi}_{1} & =v_{1}\left(-\frac{1}{L_{1}} \tan \delta-\frac{1}{L_{2}} \sin \phi_{1}-\frac{C_{1} \cos \phi_{1}-D \sin \phi_{1}}{L_{1} L_{2}} \tan \delta\right) \\
& +u_{2} \frac{1}{L_{2}} \cos \phi_{1}, \\
\dot{\phi}_{2} & =v_{1}\left(\frac{1}{L_{2}} \sin \phi_{1}+\frac{C_{1} \cos \phi_{1}-D \sin \phi_{1}}{L_{1} L_{2}} \tan \delta\right. \\
& -\frac{1}{L_{3}} \cos \phi_{1} \sin \phi_{2}+\frac{C_{1} \sin \phi_{1}+D \cos \phi_{1}}{L_{1} L_{3}} \tan \delta \sin \phi_{2} \\
& \left.+\frac{C_{2}}{L_{2} L_{3}} \sin \phi_{1} \cos \phi_{2}+\frac{C_{2}\left(C_{1} \cos \phi_{1}-D \sin \phi_{1}\right)}{L_{1} L_{2} L_{3}} \tan \delta \cos \phi_{2}\right) \\
& -u_{2}\left(\frac{1}{L_{2}} \cos \phi_{1}+\frac{1}{L_{3}} \sin \phi_{1} \sin \phi_{2}+\frac{C_{2}}{L_{2} L_{3}} \cos \phi_{1} \cos \phi_{2}\right),
\end{aligned}
$$

$$
\begin{aligned}
\dot{\delta} & =u_{1}, \\
\dot{D} & =u_{2},
\end{aligned}
$$

where $v_{3}$ is the speed of the semitrailer, given by

$$
\begin{aligned}
v_{3} & =v_{1} \cos \phi_{1} \cos \phi_{2}\left(1-\frac{C_{1}}{L_{1}} \tan \phi_{1} \tan \delta-\frac{D}{L_{1}} \tan \delta\right. \\
& \left.+\frac{C_{2}}{L_{2}} \tan \phi_{1} \tan \phi_{2}+\frac{C_{2}}{L_{2}} \frac{C_{1}-D \tan \phi_{1}}{L_{1}} \tan \phi_{2} \tan \delta\right) \\
& +u_{2}\left(\sin \phi_{1}-\frac{C_{2}}{L_{2}} \cos \phi_{1} \sin \phi_{2}\right) .
\end{aligned}
$$

By denoting the state and the control signals by $x=$ $\left[x_{3}, y_{3}, \theta_{3}, \phi_{1}, \phi_{2}, \delta, D\right]^{\prime}$ and $u=\left[u_{1}, u_{2}\right]^{\prime}$ respectively, the equations (1) can compactly be written as

$$
\frac{\partial x}{\partial t}=f_{t}\left(x(t), u(t), v_{1}(t)\right) \text {. }
$$

\section{B. Distance dependent model}

The kinematic model (3) includes the vehicle speed. Since the speed is decided by the driver at all times, it is not possible to derive a control law from this. However, due to the structure of the model, where each term has a factor of $v_{1}$, it is possible to change the dependent variable from time, $t$, to distance driven, $s$, and thereby eliminate the speed from the model. This is done by adopting the transformation

$$
\frac{\partial x}{\partial t}=\frac{\partial x}{\partial s} \frac{\partial s}{\partial t}=\frac{\partial x}{\partial s} v_{3} .
$$

The control signals are consequently changed to

$$
\begin{aligned}
& u_{1 s}=\frac{\partial \delta}{\partial s} \\
& u_{2 s}=\frac{\partial D}{\partial s}
\end{aligned}
$$

i.e. $u_{1}=u_{1 s} v_{3}$ and $u_{2}=u_{2 s} v_{3}$.

The complete distance dependent model equations are not reported here for the lack of space. However, the resulting model can be compactly written as

$$
\frac{\partial x}{\partial s}=f_{s}\left(x(s), u_{s}(s)\right),
$$

where $f_{s}$ can be easily derived from $f_{t}$.

\section{CAlCUlation OF DESIRED STATE TRAJECTORIES}

The control algorithm, described in section IV, requires the desired state trajectories along a given path. This is used both to linearize the model around the desired path and as a feed forward control action.

The starting point is a desired path in an euclidean space, $\gamma_{d}$. There is a wide variety of path planners available in literature. The path planner approach presented in [5] has been used in this paper. The idea underlying this path planning approach is to build up a path with continuous curvature, from a combination of straight lines, clothoids and circular arcs, in order to account for the nonholonomic properties of the vehicle.

From the desired path, $\gamma_{d}$, the desired state trajectory

$$
x_{d}=\left[\phi_{1 d}, \phi_{2 d}, \delta_{d}, D_{d}\right]^{\prime}
$$


as well as the desired control signals

$$
u_{d}=\left[u_{1 d}, u_{2 d}\right]^{\prime}
$$

can be calculated as functions of $s$. The method used to calculate these state trajectories is outlined in this section.

Calculating $\delta_{d}$ and $D_{d}$ is a complex task, with many possible solutions. The approach chosen in this paper is to use the coupling actuator to eliminate the swing out effect caused by the off axle hitching $\left(C_{n} \neq 0\right)$, while the steering angle is used to track the path (considering the motion of the coupling).

Optimization approaches could be used to solve the problem of calculating $\delta_{d}$ and $D_{d}$. However, heavy computational resources would be required. Hence an approximate method has been developed, which is described in the following sections.

1) Steering angle: The solution to the problem of finding the steering angle, $\delta_{d}$, corresponding to the desired path is inspired by [4], where the real vehicle is approximated by a "ghost vehicle". The "ghost vehicle" is simpler than the real vehicle, but has the same behaviour at steady state and a similar transient behaviour. Here the ghost vehicle is chosen as a truck-trailer combination with on-axle hitching, as shown in Fig. 3. As shown below, on-axle hitching allows direct calculation of the desired steering angle.

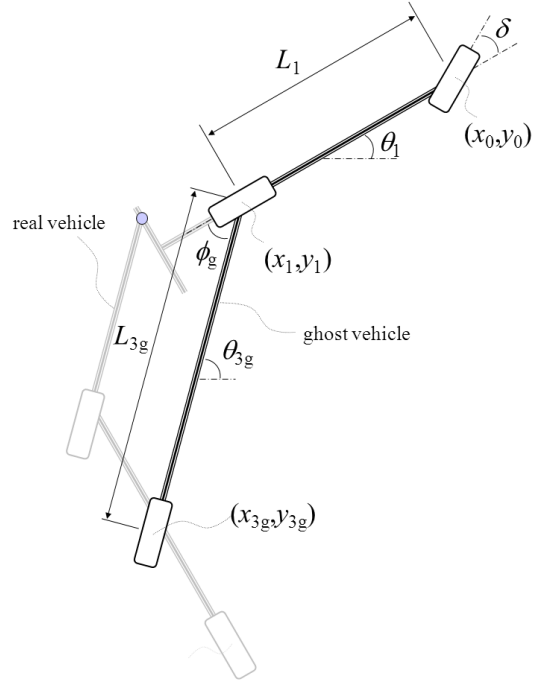

Fig. 3. "Ghost vehicle" (in black), approximating the real vehicle combination (in grey).

Denote the steady state turning radius of the semitrailer for the real and the ghost vehicle as $R_{3}$ and $R_{3 g}$. From geometry, the turning radii of the rear axle of the truck for both vehicles are

$$
\begin{aligned}
R_{1} & =\sqrt{R_{3}^{2}+L_{2}^{2}+L_{3}^{2}-C_{1}^{2}-C_{2}^{2}}, \\
R_{1 g} & =\sqrt{R_{3 g}^{2}+L_{3 g}^{2}} .
\end{aligned}
$$

Since $R_{1}=R_{1 g}$ must hold, it follows that

$$
L_{3 g}=\sqrt{L_{3}^{2}+L_{2}^{2}-C_{1}^{2}-C_{2}^{2}} .
$$

Moreover, to obtain the same turning radii for the front axle of both the real and ghost trucks, the truck wheelbases should be equal, $L_{1 g}=L_{1}$.

For the ghost vehicle it is possible to find analytic expressions for the desired states along the path. First, the orientation of the semitrailer is related to the desired curvature $\kappa_{d}$ according to

$$
\frac{\partial \theta_{3 d}}{\partial s}=\kappa_{d}
$$

Hence, integrating the curvature over the path, gives $\theta_{3 d}$ as (which by construction equals $\theta_{3 g d}$ ),

$$
\theta_{3 d}(s)=\int_{0}^{s} \kappa_{d}(\tilde{s}) \mathrm{d} \tilde{s} .
$$

Based on vehicle geometry, the desired articulation angle $\phi_{g d}$ can be calculated as

$$
\phi_{g d}(s)=-\arctan \left(L_{3 g} \kappa_{d}(s)\right),
$$

and the desired steering angle as

$$
\delta_{d}=\arctan \frac{L_{1}\left(\kappa_{d}(s)+L_{3 g}^{2} \kappa_{d}^{3}(s)+L_{3 g} \kappa_{d}^{\prime}(s)\right)}{\left(1+L_{3 g}^{2} \kappa_{d}^{2}(s)\right)^{3 / 2}} .
$$

By applying the steering input $\delta_{d}$ in (14) to the real vehicle, the vehicle closely follows the desired path. In particular, at steady state there is no error, whereas in the transient there will be a small difference. This is shown in Fig. 4(a) through a simulation, where the paths followed by the axles, $\gamma_{i}$, are plotted together with the desired path.

Moreover, the desired yaw angle of the truck $\theta_{1 d}$ is easily found as

$$
\theta_{1 d}=\theta_{3 d}-\phi_{g d}
$$

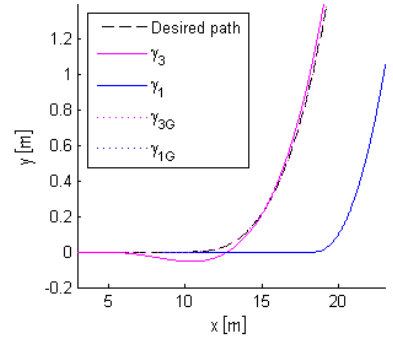

(a) Without moving the coupling.

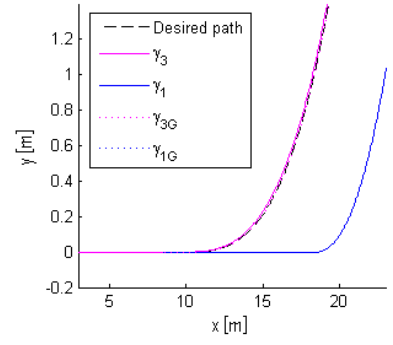

(b) With moving the coupling.
Fig. 4. Tracks of the ghost vehicle and the real vehicle when going from a straight line to a circular arc with curvature $0.05 \mathrm{~m}^{-1}$, corresponding to a steering angle of around $23^{\circ}$. When not actuating the coupling, an offset of around $5 \mathrm{~cm}$ can be seen on the semitrailer in the transient, due to the off-axle hitching. In steady state the tracks are identical. When actuating the coupling the swing out in the transient is eliminated. 


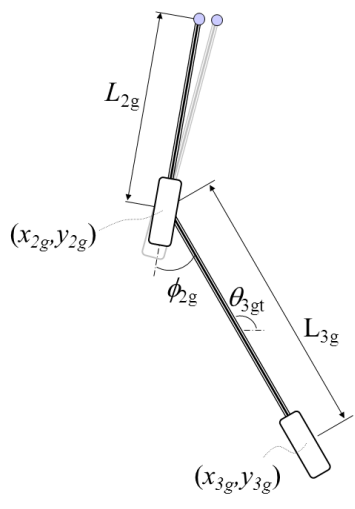

Fig. 5. "Ghost" dolly-semitrailer, approximating the real ones. The ghost trailer has the same dimensions as the real one, whereas the dolly is slightly shorter, resulting in on-axle hitching $\left(C_{2 g}=0\right)$.

2) Coupling position and state trajectories: The desired coupling position $D_{d}$ is calculated in a similar way as for $\delta_{d}$ in (14). The dolly-semitrailer is again approximated by a ghost vehicle with on-axle hitching, as in Fig. 5. To give the ghost vehicle the same length as the real vehicle, the dimensions of the ghost vehicle were chosen as

$$
\begin{aligned}
& L_{3 g t}=L_{3}, \\
& L_{2 g t}=L_{2}+C_{2} .
\end{aligned}
$$

This ghost approximation introduces an error at both the transient and steady state. But, since $C_{2}$ is very small compared to $L_{2}$ and $L_{3}$, the error is small.

By this approximation the second desired articulation angle $\phi_{2 d}$ is calculated as

$$
\phi_{2 d}(s)=\arctan \left(-L_{3 g t} \kappa_{d}(s)\right) .
$$

The angle $\phi_{1 d}$ is obtained from

$$
\phi_{1 d}=\theta_{3 d}-\phi_{2 d}-\theta_{1 d},
$$

where $\theta_{1 d}$ is given by equation (15),

The position of the coupling of the second ghost vehicle is defined throughout the whole path by

$$
\begin{aligned}
& x_{c d}=x_{3 d}+L_{3 g t} \cos \theta_{3 d}+L_{2 g t} \cos \left(\theta_{3 d}-\phi_{2 d}\right), \\
& y_{c d}=y_{3 d}+L_{3 g t} \sin \theta_{3 d}+L_{2 g t} \sin \left(\theta_{3 d}-\phi_{2 d}\right) .
\end{aligned}
$$

The path that the truck follows is also defined by the desired steering angle. Since the coupling naturally has to be attached to the truck, it is thereby possible to numerically find the desired lateral position of the coupling, $D_{d}$.

The same maneuver as in Fig. 4(a) has been simulated with a reference signal for the coupling position $D_{d}$. The results are shown in Fig. 4(b). There it can be seen that the "swing out" caused by the off axle hitching is almost completely removed. The downside with the approximation of on-axle hitching $\left(C_{2 g}=0\right)$ is a non-zero steady state error, in this case about $10 \mathrm{~cm}$. Note that this is a quite sharp turn and the error is expected to be smaller for less sharp turns. Also note that this steady state error is easier for the feedback controller to compensate for, compared to the transient error in Fig. 4(a).
3) Control signals: The feed forward control signals are simply calculated as

$$
\begin{aligned}
& u_{1 d}=\frac{\partial \delta_{d}}{\partial s} \\
& u_{2 d}=\frac{\partial D_{d}}{\partial s}
\end{aligned}
$$

\section{Control StRategy}

The control strategy chosen for solving the considered maneuvering assistance problem is a Linear Quadratic (LQ) feedback controller together with feed-forward action. The LQ gain is calculated by linearizing an offset model. The offset model, described in Section IV-A, is obtained from (6) through a change of state variables and describes the deviation of the vehicle state from the desired state trajectories calculated as in section III.

\section{A. Offset model}

Consider the state vector

$$
x_{o s}=\left[l_{o s}, \theta_{3 o s}, \phi_{1 o s}, \phi_{2 o s}, \delta_{o s}, D_{o s}\right]^{\prime},
$$

obtained from the state vector in (6) through an appropriate change of variables. In particular, as shown in Fig. $6, l_{o s}$ and $\theta_{3 o s}$ are the lateral and the heading deviations of the semitrailer from the desired path respectively (where $\theta_{3 d}$ is the desired path orientation). The remaining states in (21) are the deviations from the desired state trajectory calculated in section III.

The control signals are defined similarly by

$$
u_{o s}=\left[u_{1 o s}, u_{2 o s}\right]^{\prime}
$$

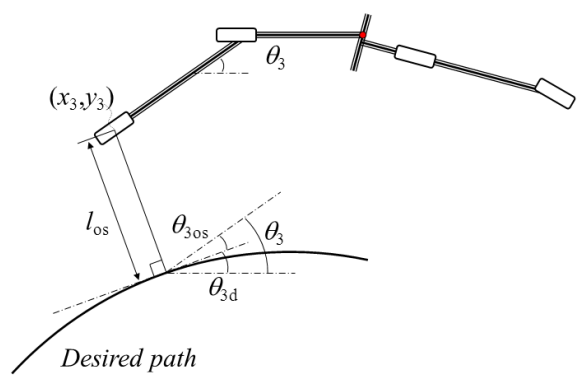

Fig. 6. The offset states are the difference between the actual states and the nominal states obtained when the vehicle follows the desired path. Here the lateral and heading offsets of the trailer are shown.

The offset model can compactly be written as

$$
\frac{\partial x_{o s}}{\partial s}(s)=f_{o s}\left(x_{o s}(s), u_{o s}(s), x_{d}(s), u_{d}(s)\right)
$$

where $x_{d}(s)$ and $u_{d}(s)$ are defined as in (7) and (8). 


\section{B. Linear model}

The kinematic model (6) can be linearized around the desired state trajectory by equivalently linearizing the model (23) around the origin. The obtained linearized model is

$$
\frac{\partial x_{o s}}{\partial s}(s)=A(s) x_{o s}(s)+B(s) u_{o s}(s),
$$

with $A$ and $B$ defined as follows

$$
\begin{gathered}
A=\left[\begin{array}{cccccc}
0 & a_{12} & 0 & 0 & 0 & 0 \\
0 & 0 & a_{23} & a_{24} & a_{25} & a_{26} \\
0 & 0 & a_{33} & 0 & a_{35} & a_{36} \\
0 & 0 & a_{43} & a_{44} & a_{45} & a_{46} \\
0 & 0 & 0 & 0 & 0 & 0 \\
0 & 0 & 0 & 0 & 0 & 0
\end{array}\right] \\
B=\left[\begin{array}{cc}
0 & 0 \\
0 & b_{22} \\
0 & b_{32} \\
0 & b_{42} \\
1 & 0 \\
0 & 1
\end{array}\right]
\end{gathered}
$$

The expressions for the matrix entries are complex and omitted here. The entries of $\mathrm{A}$ and $\mathrm{B}$ are all functions of $x_{d}$ and $u_{d}$ as shown in (27), where the $s$ argument is dropped.

$$
\begin{aligned}
a_{i j} & =g_{i j}\left(\phi_{1 d}, \phi_{2 d}, \delta_{d}, D_{d}, u_{1 d}, u_{2 d}\right) \\
b_{i j} & =h_{i j}\left(\phi_{1 d}, \phi_{2 d}, \delta_{d}, D_{d}, u_{1 d}, u_{2 d}\right)
\end{aligned}
$$

The characteristic polynomial of the system is

$$
\operatorname{det}(s I-A)=s^{4}\left(s-a_{33}\right)\left(s-a_{44}\right) .
$$

It is clear that the system has four poles in the origin and two poles in $a_{33}$ and $a_{44}$. These values have been plotted in Fig. 7 when the vehicle moves forward along steady state circles of different radii. Thereby it can be concluded that in forward driving the poles are negative, hence the system is stable. When driving backward, the poles have the same magnitude but change sign, becoming positive and hence the system is unstable. This can be confirmed by everyday experience of reversing a trailer.

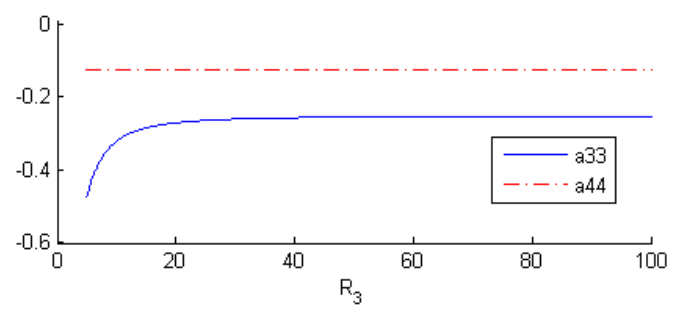

Fig. 7. $a_{33}$ and $a_{44}$ as functions of the radius of the desired path, for forward driving. For reversing, $a_{33}$ and $a_{44}$ have opposite signs.

In Fig. 7 it can also be seen that $a_{44}$ is almost constant and $a_{33}$ does not vary much with (reasonable) turning radii. The same actually holds for all the elements of the matrices A and $\mathrm{B}$. Hence it is reasonable to linearize the model around a straight line only.

\section{Control algorithm}

The steps of the control algorithm are outlined in Fig. 8. First the initial and target states are provided to the path planner, which calculates a desired path $\gamma_{d}$ in the euclidean space. From this path and the "ghost" approximation described in section III, the desired states $x_{d}$ and the feed forward signals $u_{d}$ are found throughout the path. These states are then used to find the linear offset model presented in section IV-B. The matrices $A$ and $B$ of the linearized model are finally used to solve the Riccati equation, giving the feedback gain $L$ of the LQ controller. These steps are performed once, before starting the maneuver. Then the gains, together with the feed forward signals and euclidean path, are sent to the control loop running throughout the maneuver.

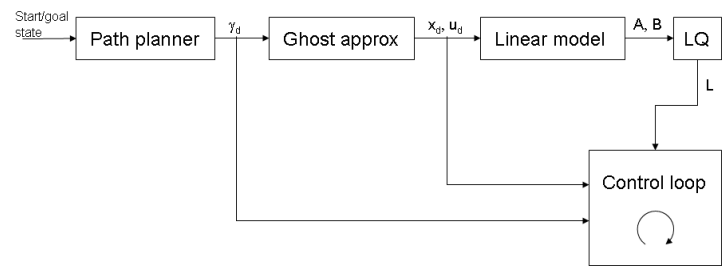

Fig. 8. Steps of the control algorithm. The top parts are ran once before starting the motion and the control loop.

The control scheme running throughout the motion is shown in Fig. 9. Starting from the vehicle, the state $x$ is measured. In an interpolation step the closest point of the desired path $\gamma_{d}$ is found. The states of this point are compared to the desired ones and the offset states $x_{o s}$ are calculated. Also the distance driven along the path $s$ is found. The offsets are then multiplied by $-L$ to calculate the feedback component of the control signal $u_{o s}$. This is then added to the feed forward signal $u_{d}$, found through the ghost approximation and the travelled distance. This control signal $u_{s}$ is the partial derivate of the steering angle and coupling position with respect to driven distance $s$. Hence, according to (5), $u_{s}$ needs to be multiplied by the current speed $v_{3}$ before being fed to the actuators of the vehicle.

As shown in Fig. 9, the integral of $l_{o s}$ has also been added to the state vector. This is to compensate for the steady state errors originating from the model approximations.

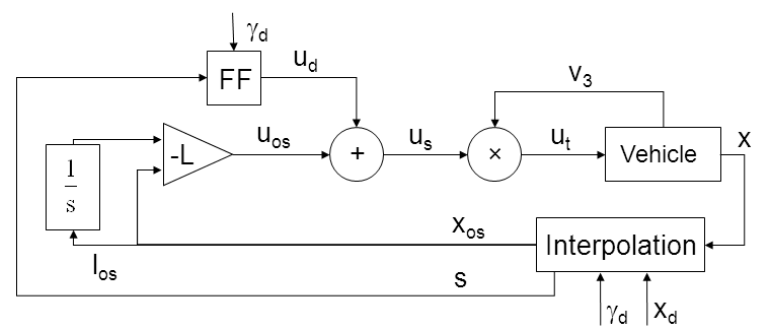

Fig. 9. Control loop running throughout the motion. 


\section{RESULTS}

Simulations have been performed to test the controller presented in Section IV. As plant model the Virtual Truck Model (VTM), presented in section II, has been used.

\section{A. Simulation parameters}

The vehicle used in simulations is, as mentioned earlier, a standard $25.25 \mathrm{~m}$ Nordic Combination, shown in Fig. 1. The equivalent wheelbases in Fig. 2 are

$$
\begin{array}{ll}
L_{1} & 5.668 \mathrm{~m} \\
L_{2} & 3.960 \mathrm{~m} \\
L_{3} & 7.876 \mathrm{~m} \\
C_{1} & 2.435 \mathrm{~m} \\
C_{2} & -0.110 \mathrm{~m}
\end{array}
$$

The maximum steering angle is $\pm 36^{\circ}$ and the maximum lateral coupling position is $\pm 0.8 \mathrm{~m}$. The speed $v_{1}$ is set to $1 \mathrm{~m} / \mathrm{s}$ in the simulations presented next (note that the the only way the speed affects the control loop is through the feedback of $v_{3}$ ).

The weight matrices in the LQ controller have been chosen as

$$
\begin{aligned}
& Q=I, \\
& R=2 I,
\end{aligned}
$$

where $I$ is the identity matrix.

\section{B. Tight 90 degree turn with cusps}

The first considered test case is a tight 90 degree turn. In this context, "tight" means that it is not possible to perform the turn in one move but a more complex maneuver is required. In fact this covers all the basic maneuvers that could be planned by the path planner and is therefore a good test case of the algorithm. The vehicle starts from the origin with heading angle of $90^{\circ}$, while the goal position is $(15,-10)$ with heading angle of $180^{\circ}$, as shown in Fig. 10 .

Fig. 10 also shows the path planned by the path planner. As mentioned earlier, the planner calculates a desired path for the semitrailer. Here the path starts with reversing along a clothoid and then along a circular arc. Then there is a cusp, i.e., a change of motion direction, before the vehicle starts moving forward along a clothoid, then a straight line and a clothoid before the next cusp. The final reversing part consists of a circular arc and a clothoid.

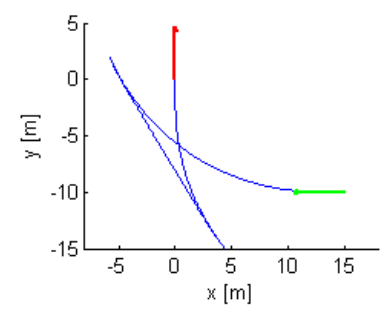

Fig. 10. First test case consisting of a tight $90^{\circ}$ turn. The red arrow shows the start position and heading, whereas the green arrow shows the goal position and heading. The blue line is the path planned by the path planner.
Fig. 11 shows the paths followed by the the front axle of the truck, $\gamma_{0}$, and the semitrailer axle, $\gamma_{3}$, during the turn. As can be seen here, and more clear in Fig. 12, the semitrailer follows the desired path, $\gamma_{d}$, closely. Also note how the front of the truck moves to allow the semitrailer to follow the desired path.

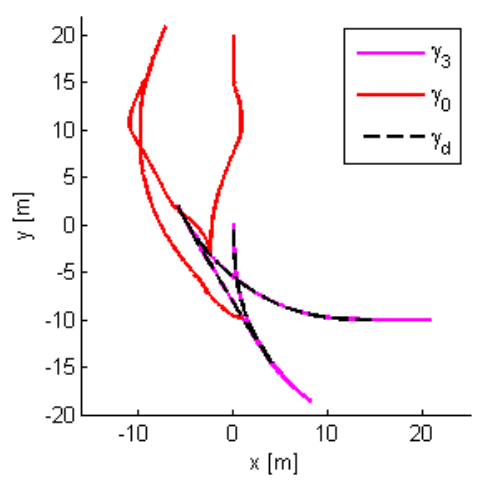

Fig. 11. Figure showing how the front axle of the truck and the semitrailer axle move during the tight $90^{\circ}$ turn.

Fig. 12 shows lateral and heading offsets, $l_{\text {os }}$ and $\theta_{3 o s}$, of the semitrailer from the desired path. There it can be seen that the trailer deviates at most, $17 \mathrm{~mm}$ from the path. Not surprisingly the largest errors occur in the transients between the straight lines and the arcs of circles, i.e. in the clothoids. This is because there the vehicle is no longer in steady state and needs to change its articulation angles. Also the approximations in section III introduce a small error here. The heading error is also small, below $0.0086 \mathrm{rad}\left(0.49^{\circ}\right)$ during the maneuver.

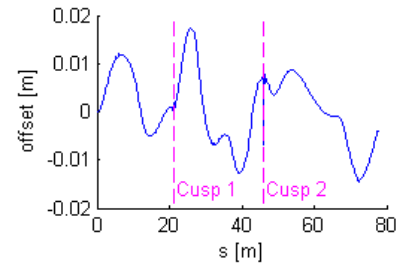

(a) Lateral offset $l_{\text {os }}$.

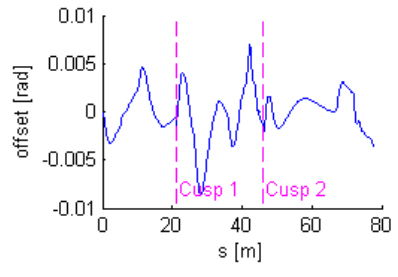

(b) Heading offset $\theta_{3 o s}$.
Fig. 12. Lateral and heading offset, $l_{o s}$ and $\theta_{3 o s}$, of the semitrailer during the maneuver shown in Fig. 10.

Fig. 13 shows how the actuators work during the maneuver. There it can be seen that $\delta$ and $D$ are close to the desired ones calculated through the ghost approximation in section III. In Fig. 13(b), four "sine like" motions of the coupling can be seen, e.g. to the far right. These motions occur in the transients (clothoids) where they try to eliminate the swing out, described in section III. Similarly, seen in Fig. 13(a), the steering angle varies most in the transients and is almost constant in steady state.

\section{Speed}

The same maneuver as in section V-B was simulated at higher speeds, see Fig. 14. At $2 \mathrm{~m} / \mathrm{s}$ the maximum lateral 


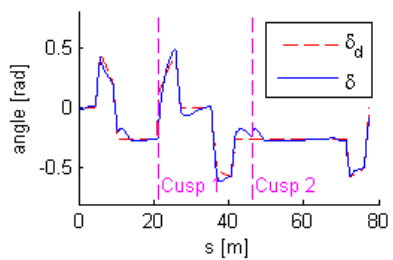

(a) Steering angle.

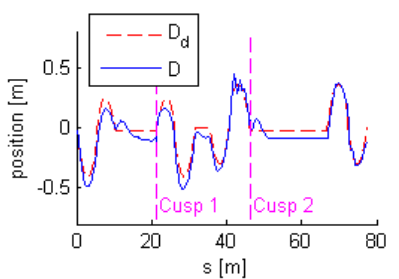

(b) Coupling position.
Fig. 13. Feed forward and actual steering angle and coupling position during the maneuver in Fig. 10.

error was $79 \mathrm{~mm}$ and at $3 \mathrm{~m} / \mathrm{s}$ the maximum lateral error grew to $170 \mathrm{~mm}$. Then also the actuators saturate for a short time at some points of the maneuver. The main reason for the larger errors are naturally that the approximations used to derive the vehicle model are less accurate at higher speeds.

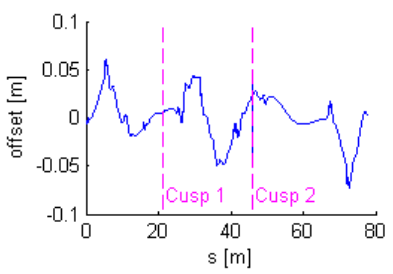

(a) $2 \mathrm{~m} / \mathrm{s}$

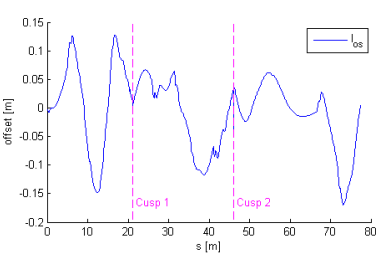

(b) $3 \mathrm{~m} / \mathrm{s}$
Fig. 14. Lateral offset from the path, $l_{o s}$, of the semitrailer during the maneuver shown in Fig. 10 in a speed of 2 and $3 \mathrm{~m} / \mathrm{s}$.

\section{Robustness}

The previous test case of a tight $90^{\circ}$ turn covers the most difficult maneuvers that could be given by the path planner. The algorithm has been further tested with initial conditions far from the path. Two tests are shown next, where the planned path is a $50 \mathrm{~m}$ straight reversal. In the first case the vehicle starts parallel to the path, $0.5 \mathrm{~m}$ to the left. In the second case the vehicle starts on the path, but with a heading offset of $10^{\circ}$. Fig. 15 shows how the lateral errors evolve. The vehicle clearly converges within about $40 \mathrm{~m}$ in both cases, which is reasonable considering that the vehicle itself is $25 \mathrm{~m}$ long.

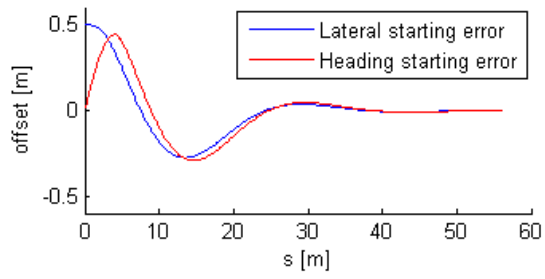

Fig. 15. Lateral offset for a planned straight reversal, letting the vehicle start with an initial error. In the first case the initial error is a lateral offset of $0.5 \mathrm{~m}$, whereas in the second case the initial error is a heading offset of $10^{\circ}$.

\section{Vi. Conclusions}

A maneuvering assistance system has been presented in this paper. The assistance plans a feasible path, considering the nonholonomic motion constraints of the vehicle combination. The path is then followed by an LQ controller, calculating the steering angle and coupling point position. Simulation results show that the control algorithm tracks the planned paths closely at low speeds. The proposed controller is also proven to be robust with respect to initial offsets from the desired path.

\section{ACKNOWLEDGMENTS}

The authors would like to thank Leo Laine and Kristoffer Tagesson at Volvo Group Trucks Technology for valuable discussions and feedback.

\section{REFERENCES}

[1] C. Altafini, A. Speranzon, and B. Wahlberg. A feedback control scheme for reversing a truck and trailer vehicle. Robotics and Automation, IEEE Transactions on, 17(6):915-922, 2001.

[2] A. Astolfi, P. Bolzern, and A. Locatelli. Path-tracking of a tractortrailer vehicle along rectilinear and circular paths, a lyapunovbased approach. Robotics and Automation, IEEE Transactions on, 20(1):154-160, 2004.

[3] P. Bolzern, R. M. DeSantis, and A. Locatelli. An input-output linearization approach to the control of an n-body articulated vehicle. Journal of Dynamic Systems, Measurement, and Control, 123(3):309_ 317, 2001.

[4] P. Bolzern, R.M. DeSantis, A. Locatelli, and D. Masciocchi. Pathtracking for articulated vehicles with off-axle hitching. Control Systems Technology, IEEE Transactions on, 6(4):515-523, 1998.

[5] T. Fraichard and A. Scheuer. From reeds and shepp's to continuouscurvature paths. Robotics, IEEE Transactions on, 20(6):1025-1035, 2004

[6] S.-G. Kong and B. Kosko. Adaptive fuzzy systems for backing up a truck-and-trailer. Neural Networks, IEEE Transactions on, 3(2):211223, 1992.

[7] D. Nguyen and B. Widrow. The truck backer-upper: an example of self-learning in neural networks. Neural Networks, 1989. IJCNN. International Joint Conference on, 2:357-363, 1989.

[8] Hans B. Pacejka. Tire and vehicle dynamics. Butterworth-Heinemann, Oxford, 2006.

[9] M. Sampei, T. Tamura, T. Kobayashi, and N. Shibui. Arbitrary path tracking control of articulated vehicles using nonlinear control theory. Control Systems Technology, IEEE Transactions on, 3(1):125-131, 1995.

[10] Christopher B Winkler. Simplified analysis of the steady-state turning of complex vehicles. Vehicle System Dynamics, 29(3):141-180, 1998. 